\title{
Increased Energy Efficiency via Delay-Tolerant Transmissions in Cognitive Radio Networks
}

\author{
Bi Zhao \\ Centre for Telecommunications Research \\ King's College London, London, UK \\ E-mail: bi.zhao@kcl.ac.uk \\ Vasilis Friderikos \\ Centre for Telecommunications Research \\ King's College London, London, UK
}

Received: February 15, 2013

Accepted: June 18, 2013

Published: June 30, 2013

DOI: 10.5296/npa.v5i2.3265

URL: http://dx.doi.org/10.5296/ npa.v5i2.3265

\begin{abstract}
We are currently witnessing the emergence of two important trends in wireless networks, namely the increased usage of Internet like applications, many of them which are delay tolerant, and cognitive radio techniques. In this paper, the focus is on how to capitalize the delay tolerance of various applications (such as email, Peer to Peer networks, social networking $\&$ operating system updates and file transfers to mention just a few) to reduce the energy consumption in cognitive networks. Since it becomes feasible to estimate the Primary Users (PU) connections for the Secondary Users (SU) by contacting a trusted database containing the information of PU traffic, a scheme is proposed that explicitly utilize the distribution of SU traffic loads to provide load-balancing. By modeling the problem under investigation as an $\mathrm{M} / \mathrm{M} / \mathrm{K} / \mathrm{L}$ queuing system, the performance of the SUs competition is analyzed under various traffic blocking thresholds and queuing delays. To this end, by optimizing the frequency channel utilization, the number of SU connection that can be accommodated simultaneously is derived. A wide set of numerical investigations reveal how message transmission delays and the use of available white spaces can reduce the energy consumption without affecting PU traffic.
\end{abstract}

Keywords: Delay Tolerant Networking, Queuing Theory, Cognitive Radios 


\section{Introduction}

Spectrum is a fundamental resource in wireless networks since achievable data rates are linearly dependent on the available spectrum in use. It is widely acknowledged that the utilization of the spectrum can be deemed a sparse and shows significant spatio-temporal variations. Exploiting these spectrum white spaces, as they are called, has been the focus of Cognitive Radios (CRs). CR networks encompass essentially two core functionalities, namely, spectrum sensing and spectrum management [1]. Spectrum sensing functionalities are responsible to detect unused spectrum and a plethora of different methods have been considered to do so which can be broadly taxonomized in cooperative and non-cooperative techniques reflecting whether the detection is taking place by the node itself or by utilizing other nodes in the network. Spectrum management on the other hand can be considered as the decision engine (mode of operation and parameters that will be used for transmission) on how to optimize the use of spectrum based on the information available from the spectrum sensing functionalities.

In essence, $\mathrm{CR}$ networks pose the potential of improving spectrum efficiency by allowing the low-priority SUs to temporarily seek the wireless spectrum that is licensed to different organizations (PUs) [2]. As soon as the PUs emerge in the frequency channels, the SUs must vacate the licensed bands. The SU connections would be interrupted by the stochastic nature of the PU traffic. Consequently, the SUs should firstly estimate the channel availability by probability analysis based on PUs' historical traffic information or spectrum sensing. A common technique for channel estimation has been to analyze the PU traffic characteristic from available long-term observations/statistics [3]. In [4], authors design optimal sensing strategies via a model assuming that the PU transmissions are unslotted as a continuous-time Markov chain while the SUs are slotted to sense the frequency channels. On this basis, Noh et al. propose a stochastic multichannel sensing scheme based on traffic information and sensing history [5]. We assume that the SUs can achieve perfect channel estimation about the PU connections from the Base Station (BS) or other wireless providers. In accordance with the information of channel utilization, SU connections can decide which frequency channels are available to utilize without deteriorating the quality of the PU connections.

Queuing theory is a natural mathematical tool to analyze system performance by considering stochastic arrivals and departures as is the case in wireless networks. As such, queuing theory has been applied in significant volume of research efforts within CR literature. In [6], by considering a two-dimensional M/M/N/N queue, the authors have derived the mean number of the PUs and SUs with their blocking probabilities respectively. However, they focused merely on the situation where the SU connections would be immediately dropped if all frequency channels are occupied by PUs in Secondary Users Cleared (SUC) mode, and the situation that the PU and SU connections have equal priority in Secondary User Equality (SUE) mode. In our model, we assume that there are $\mathrm{M} / \mathrm{M} / \mathrm{K} / \mathrm{L}$ queue systems for the $\mathrm{SU}$ connection, in which the SU message could be buffered. A preemptive priority queuing system has been utilized to analyze the mean system dwelling time of the SU traffic and the blocking probability for real-time SU connections [7]. In [8], the authors analyze the queue lengths and average queuing delay of the SUs based on Poisson distribution of the SUs. In [9], 
a Dynamic Strategy Learning (DSL) algorithm relied on the priority queuing systems including the SUs and the PUs is proposed for the delay-sensitive multimedia applications in order to maximize the user's utility function.

Considering that an increasing number of countries are now permitting operation of cognitive radio systems in the vacant terrestrial analog TV transmission - TV White Space (TVWS), it will create new possibilities to provide better wireless broadband and multimedia services [10]. When the SUs are using 3G cellular networks within the coverage of a TVWS master, it is possible that the SUs would prefer the TVWS connection over the cellular networks in terms of cost, RF coverage, capabilities, and overall Quality of Service. The SU connection can utilize the means as sensing or contacting a trusted geospatial database that records the information regarding PUs occupation with a specific location and time duration, prior to message transmission, to determine available spectrum at a given location [11]. Moreover, when the SUs are vehicular users, they may want to build the connectivity to another wireless node in peer-to-peer or ad-hoc networks. In this scenario, to predict the future location and the path of mobility of wireless nodes is another challenging issue in the so-called White-Fi networks.

In our context, in order to capitalize the delay tolerance of applications, we develop a theoretical framework for energy efficiency schemes that can maximize spectrum utilization and SU throughput. Our approach relies on Adaptive Modulation and Coding (AMC) to dynamically change the modulation and coding scheme. Once the mobile devices are equipped with a dual or multi mode antenna that is connected to $3 \mathrm{G}$ and other networks like TVWS, the SUs could switch between these networks to seek and use any licensed spectrum bands as long as they do not cause interference to the PUs. By considering the distribution of the SU traffic load and PU connection that would be emerging stochastically, the CR system will contact a trusted database for historical information about PU traffic at a specific location and time duration so as to estimate the probability for the SU connections. Based on the estimation of PU traffic within a specialized relatively long-term duration, and the analysis of probabilities for vacant channels and time slots, an $\mathrm{M} / \mathrm{M} / \mathrm{K} / \mathrm{L}$ queuing system is devised to estimate the SU traffic capability that the system can serve simultaneously. By considering an $\mathrm{M} / \mathrm{M} / \mathrm{K} / \mathrm{L}$ queue where $\mathrm{SU}$ message compete for $\mathrm{K}$ frequency channels in $\mathrm{N}$ concentric rings with different modulation and coding schemes within the BS coverage, the traffic of the SUs can be inputted to the queue for the SU connections. If the number of SUs is large, the input traffic of the virtual queue can be modeled as a Poisson process, where L is the finite number of waiting positions for each queue. Finally, for the delay-tolerant applications, we recommend the system to delay the message transmission to an area close to the BS, thereby maximizing the throughput potential whilst reducing the overall energy cost of message transmission under several constraints.

The rest of the paper is organized as follows. In Section II, the related work on spectrum selection schemes and queuing models in CR are summarized. Section III defines the model of energy consumption over cellular networks, and formulates the optimal SU connection approximation in the queuing system of CR networks. Section IV presents numerical investigations and analysis of optimal transmission schemes. Finally, we conclude the work 
in Section V.

\section{Related Work}

\subsection{Delay Tolerant Networks}

Significant volume of research efforts have been placed on energy efficient data transmission for delay tolerant applications, especially looking at the trade-offs between transmission cost and delay tolerance over wireless networks [12] [13] [14] [15]. The work in [12] deals with the problem of packet scheduling with deadlines within a pre-defined time window [0, T). Based on that, the authors in [16] explore the energy-efficient packet transmission with individual packet delay constraints, in which a trade-off between flexible energy and delay is analyzed under various individual packet delay constraints and bandwidth efficiencies.

In [13], the authors consider a delay constraint for each packet and reveal the relationship between reliable transmission rate and QoS requirements, while a dynamic programming based algorithm is introduced to acquire throughput maximization and energy minimization according to different channel qualities of a fading channel with time constraints [14]. The work in [15] further investigates the problem of energy-delay trade-offs under dynamic traffic loads and user populations. The target-set selection problem has been studied in the emerging Mobile Social Networks for traffic offloading by delaying the delivery [17]. In [18], a framework is proposed to investigate the trade-off between the amount of offloaded traffic and the users' delay tolerance over a $3 \mathrm{G}$ network.

\subsection{Channel Selection}

There have been several prior works on dynamic spectrum access, especially channel selection schemes. In [19], a dynamic protocol is introduced for dynamic spectrum allocation (DSA) with load balancing for SUs. They assume that the PU traffic detection and SU connections blocking in these channels from the allocation game has been solved already, therefore they just focus on the load balancing of SU traffic. Their algorithms are modeled as a so-called balls and bins problem of congestion game, where balls representing the CRs are assigned to several bins representing physical channels of the radio spectrum and SUs may reassign its load in a round based fashion dynamically. The system can rapidly converge towards an approximately balanced state in which all CRs will sustain cost below a certain threshold parameter.

In [20], authors propose an effective spectrum decision scheme, which can evenly distribute the traffic load of SU connections to multiple channels, thereby reducing the average overall system time compared to the non-loadbalancing scheme. They discuss two kinds of spectrum decision schemes: one is sensing-based with the objective to determine the optimal sensed number of candidate channels for channel selection, the other is probability-based that take the traffic statistics of both PUs and SUs. In [21], the authors design a minimum collision rate algorithm and minimum handoff rate algorithm to maximize spectrum holes utilization of the PU channels for optimal SU throughput on the basis of 
satisfying constraints of collision tolerable level of PU channels. These two channel selection schemes are based on spectrum holes prediction from past observations. In [22], a opportunistic channel selection scheme is proposed with the aid of statistical traffic pattern of PU channels and traffic prediction techniques. According to the long-term statistical probability of each channel appearing idle in the next time slot, SU will firstly sense the channel with the highest average probability of being idle. Likewise, a channel selection method has been devised in [23] to analyze the most probable unoccupied channels for SU traffic. When a database receives a SU query for an unoccupied channel, it will deliver stored historical information about channel availability. The database searches the channels that have been unoccupied at previous days from that time slot to some time to future, while it ensures noise levels of the most probably vacant channels is lower than a threshold.

In [24], in order to achieve better channel utilization, SUs need to make a decision at each time slot to access if the current achievable throughput can be improved by utilizing another physical channel based on channel sensing and estimation. This channel exploitation problem can be modeled as a finite-horizon optimal stopping problem between the expected increasing data rate of SU and channel sensing cost.

\subsection{Queuing Theory in CR}

Many different facets of queuing theory have been utilized in the CR literature to analyze the different priorities schemes for PU and SU traffic. In [25], a slotted transmission system and an infinite queue are assumed for both PU and SU traffic. SUs sense the channels in each slot, and transmit a packet from the corresponding queue if detecting an idle time slot. In this context, SU link has been considered as a "transparent" relay for the PU traffic, if it does not affect the stability of the PU queue. In [26], the preemptive resume priority (PRP) M/G/1 queuing network model is developed to characterize the channel usage of CR networks. Based on this queuing model, they characterize the spectrum utilization behavior of each channel with different arrival rates and service time distributions of PUs and SUs. The research investigates the effects of spectrum hand-off delay on the extended data delivery time of the SU connections.

A significant volume of research took the approach of categorizing users into $\mathrm{K}$ priority classes. In that case, the highest priority class $C_{1}$ is always reserved for the PUs in each channel and SUs occupy the rest of $\mathrm{K}-1$ priority classes $\left(C_{2}, C_{3}, \cdots, C_{k}\right)$ [9] [27]. In [9], an $\mathrm{M} / \mathrm{G} / 1$ model is adopted and each SU maintains several physical (or logical) queues for the various frequency channels. The channel selection decisions are based on priority queuing analysis that considers the dynamic channel conditions, traffic characteristics, and the competitors' behavior. Hence, the SUs could efficiently adapt their channel selection strategies. In [27], they also model the traffic as an M/G/1 preemptive priority queue with channel conditions and one PU and multiple SUs competing for the same frequency channels. Vacating the channels for the PU traffic, SUs would sense the remained channels and time slots (spectrum holes) according to a simple First-Come-First-Serve (FCFS) rule. Moreover, they place the emphasis on the delay and throughput of the SU traffic instead of stability.

An $M / M / 1$ time-varying queuing model is introduced to generate an accurate temporal 
and frequency characterization of CR networks in [28]. In this case, the single server is assumed to be a centralized BS and spectrum occupancy and availability for SU connections is based on a statistical model. In [29], the authors design a model that PUs occupy many licensed channels with one channel allocated for SU traffic. The SU traffic subsystem is modeled as an M/M/1 queue with a processor sharing (PS) policy. Meanwhile, the SUs would sense the PU licensed channels in order to find a free channel for transmission. As a result, the traffic of PU license channels could be modeled using an $\mathrm{M} / \mathrm{M} / \mathrm{K} / \mathrm{K}$ queue. PU traffic in three different levels of priority compared to SU connections (perfect, partial and no priority) has been analyzed in [6], where an M/M/N/N queuing system is utilized to analyze PU and SU traffic of arrival process and service rates.

In [7], the preemptive priority queuing model is designed to analyze the SU traffic of CR system. The distributed non-real-time SU traffic enters a queue buffer in First-In First-Out (FIFO) manner when the server is busy with PU or earlier SU connections. In non-real-time situation, SU traffic are allowed to be buffered in queues. Meanwhile, if there is no server available, the real-time SU traffic are immediately discarded. They show the simulation results regarding system dwelling time for non-real-time SU connections and blocking probability and forced termination probability for real-time SU traffic under different SU arrival rates.

\section{System Model}

Hereafter, a single cell wireless network is considered where Adaptive Modulation and Coding (AMC) are supported. To this end we denote with $\left\{M_{R_{1}}, M_{R_{2}}, \cdots, M_{R_{N}}\right\}$ the set of available modulation and coding schemes which can be selected in essence according to the distance between the wireless device and the BS. In that respect, the cell can be envisioned as being separated into $\mathrm{n}$ concentric rings of radii $R_{i}, i=1,2, \cdots, N$ as shown in Fig. 1 . In this case, each circular region with distance $R_{i}$ to the BS corresponds to a different constellation size and coding scheme.

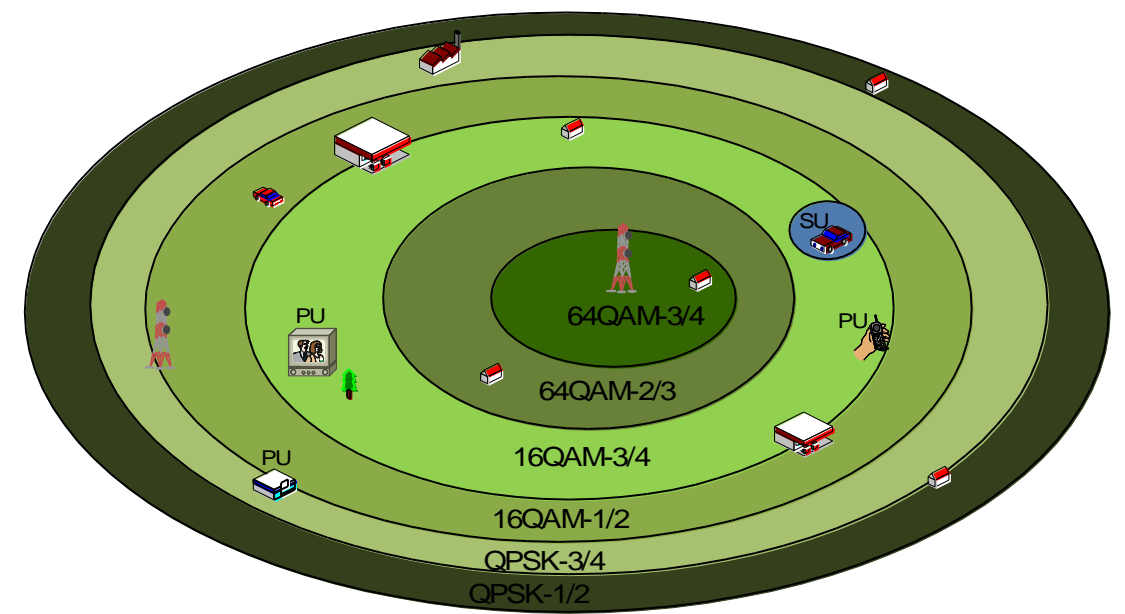

Figure 1. Graphical representation of a cell decomposed into six concentric rings corresponding to different transmission rates 
Let $\mathrm{r}$ denote the coding rate, and $r_{B S}$ be the (Euclidean) distance between the mobile node and the serving BS. The spectral efficiency (bits/s/Hz) is given by [30]:

$$
\operatorname{IEC}\left(r_{B S}\right)=r \cdot \log _{2}\left(M_{r_{B S}}\right) b i t / s / H z
$$

Concerning the energy consumption in message transmission, we identify the following main sources: (1) the electronic circuit at the nodes of SU; (2) the energy dissipated to transmit the message; and (3) the energy consumed to receive corresponding messages. The energy consumption regarding SU wireless message transmission can be formulated as in [31], shown in the equation below where $e_{d}$ represent the energy consumption of the op-amp and $\mathrm{B}$ represents the data transmission rate.

$$
\begin{aligned}
& f\left(r_{B S}\right)=\left(e_{r x}+e_{t x}+e_{d} \cdot r_{B S}^{\eta}\right) B \cdot \tau \\
& =\left\{\begin{array}{lll}
\left(e_{r x}+e_{t x}+e_{l o s} \cdot r_{B S}^{2}\right) \cdot B \cdot \tau & \text { if } & r_{B S} \leq r_{t h s} \\
\left(e_{r x}+e_{t x}+e_{m p} \cdot r_{B S}^{4}\right) \cdot B \cdot \tau & \text { if } & r_{B S}>r_{t h s}
\end{array}\right.
\end{aligned}
$$

where $e_{\text {los }}$ denotes the one bit energy consumption with the free space (line-of-sight) propagation losses (the distance to the $\mathrm{BS} r_{B S} \leq r_{t h s}$ ) and $e_{m p}$ is the energy consumed per bit in transmission in the longer-distance case that the signal is attenuated by multi-path fading (Two-ray Reflection). Let $P_{l o s}$ and $P_{m p}$ denote the transmitted signal powers in these two cases respectively, we obtain

$$
\begin{aligned}
& P_{l o s}=\frac{P_{r x}(4 \pi)^{2}}{\lambda_{w l}^{2} G_{t x} G_{r x}} \cdot r_{B S}^{2} \\
& P_{m p}=\frac{P_{r x}}{\left(h_{t x} h_{r x}\right)^{2} G_{t x} G_{r x}} \cdot r_{B S}^{4}
\end{aligned}
$$

where $\lambda_{w l}$ is the wavelength, $h_{t x} / h_{r x}$ is the transmit/receive antenna height, and $G_{t x} / G_{r x}$ is the transmitter/receiver antenna gain. Therefore, the energy consumption for transmission is given by:

$$
f\left(r_{B S}, t\right)=\left(e_{r x}+e_{t x}\right) F+\sum_{t=\tau_{k}}^{\tau_{k+m}} P_{l o s}\left(\text { or } \quad P_{m p}\right) \cdot t
$$

where $\tau_{k+m}$ is the time slot that finish the message transmission

Let $\Gamma \in\left\{\tau_{k}, \tau_{k+1}, \tau_{k+2}, \cdots, \tau_{k+m}\right\}$ denote the time units for message transmission in one circular region, where $\tau_{k}$ is the time unit to launch the message transmission, and $\tau_{k+m}$ is the time unit that complete the transmission. In each time unit, one message block will be 
transmitted to the serving BS. Consequently, the length of time units in each ring should be different due to the different throughput achieved in each of the different circular areas of the cell.

Within cognitive radio (CR) networks, the SU transmission is trying to explore white space of wireless spectrum while at the same time avoiding interfering with the PU traffic. For the purpose of avoiding PU transmission, as a SU, the fundamental assumption is that wireless nodes in vehicles use spectrum sensing or query the database which maintains information about the available channels for the details of the local radio environment.

\subsection{Optimal Transmission Time of SUs}

In the proposed model as detailed hereafter we assume that wireless nodes gather PU traffic information from a historical database in order to predict over a short-term the traffic pattern of PUs. In the database server, there are two types of information about primary channels. One is the 24-hours traffic characteristics of different channels in several months [3], the other is the noise power level in different channels that updated by SU devices constantly. Based on the above framework, a SU has to send a query to database server for the available channel to transmit. According to the available channel information at the same time slots in previous days and long-term statistics regarding channel availabilities, the database server will certificate the noise power levels by comparing to a threshold. Then the best candidate channels for the inquired SU will be determined.

As there is significant previous research regarding the prediction of PU connections arrival rate and holding times, we aim to utilize such results and design an algorithm for SU connections according to the periodicity of PU traffic pattern. In this scenario, we consider the time horizon for the estimation to be relatively long term (such as for example in terms of hours) instead of short term (fraction of a second), that is, the prediction of PU traffic is stable and unchangeable in a relative long-term time. Therefore, under the assumption of the probability-based channel selection scheme [20], the SU will select its operating channel from all of the $\mathrm{M}$ candidate channels to achieve some form of load-balancing based on the stochastic traffic prediction, such as the arrival rates and service times. Applying the Probability-based Scheme theory [20], the average system time S is given by,

$$
E[S]=E[W]+E[T]
$$

where $\mathrm{W}$ denotes the waiting (queuing) time and $\mathrm{T}$ presents the extended data transmission time. Then, let $X_{p}^{(k)}$ and $X_{s}^{(k)}$ be the extended service time of PU and SU respectively due to the missed detection and false alarm probabilities. $\lambda_{p}^{(k)}$ and $\lambda_{s}$ are the average arrival rates of the PU connections at channel $\mathrm{k}$ and the SU connections. $\rho_{p}^{(k)}$ and $\rho_{s}^{(k)}$ are the busy probabilities resulting from the PU and SU connections at channel k.

$$
E[T]=\sum_{k=1}^{K} p_{p b}^{(k)} \frac{E\left[\tilde{X}_{s}^{(k)}\right]}{1-\rho_{p}^{(k)}}
$$




$$
\begin{aligned}
& E[W]=\sum_{k=1}^{K} p_{p b}^{(k)} \frac{\frac{1}{2} \lambda_{p}^{(k)} E\left[\left(\tilde{X}_{p}^{(k)}\right)^{2}\right]+\frac{1}{2} p_{p}^{(k)} \lambda_{s} E\left[\left(\tilde{X}_{s}^{(k)}\right)^{2}\right]}{\left(1-\rho_{p}^{(k)}\right)\left(1-\rho_{p}^{(k)}-\rho_{s}^{(k)}\right)} \\
& \rho_{p}^{(k)}=\lambda_{p}^{(k)} E\left[\tilde{X}_{p}^{(k)}\right] \\
& \rho_{s}^{(k)}=\lambda_{s}^{(k)} E\left[\tilde{X}_{s}^{(k)}\right]
\end{aligned}
$$

where the vector $p_{p b}=\left(p^{(1)}, p^{(2)}, \cdots, p^{(k)}, \cdots p^{(K)}\right)$ presents the set of distribution probabilities of candidate channels. We utilize a probability-based scheme to find the optimal probability vector $p_{p b}$ in order to balance the PU and SU traffic load among multiple channels with the constraint that $p^{(1)}+p^{(2)}+\cdots+p^{(K)}=1$. Moreover, this optimal $p_{p b}$ vector can maximize the transmission time in each time slot in order to accommodate more SU traffic. On the basis of this optimal vector, the SUs could directly access the preselected channel by estimating the PU traffic characteristics from historical stored data.

From Fig. 2, we can observe that when the SU traffic is relatively light, i.e., the arrival rate being 0.01, the SU traffic prefers to select channel 1 as their transmission frequency channel which has the lightest PU traffic. However, when the SU arrival rate becomes higher as the arrival rate is 1 , all of the channels seem to be identical for the SU traffic, and the SU connections don't have any priority over the candidate channels for message transmission. In our model, it is assumed that the average arrival rates of the SU connections are under a threshold which restricts the SU traffic for better QoS service.

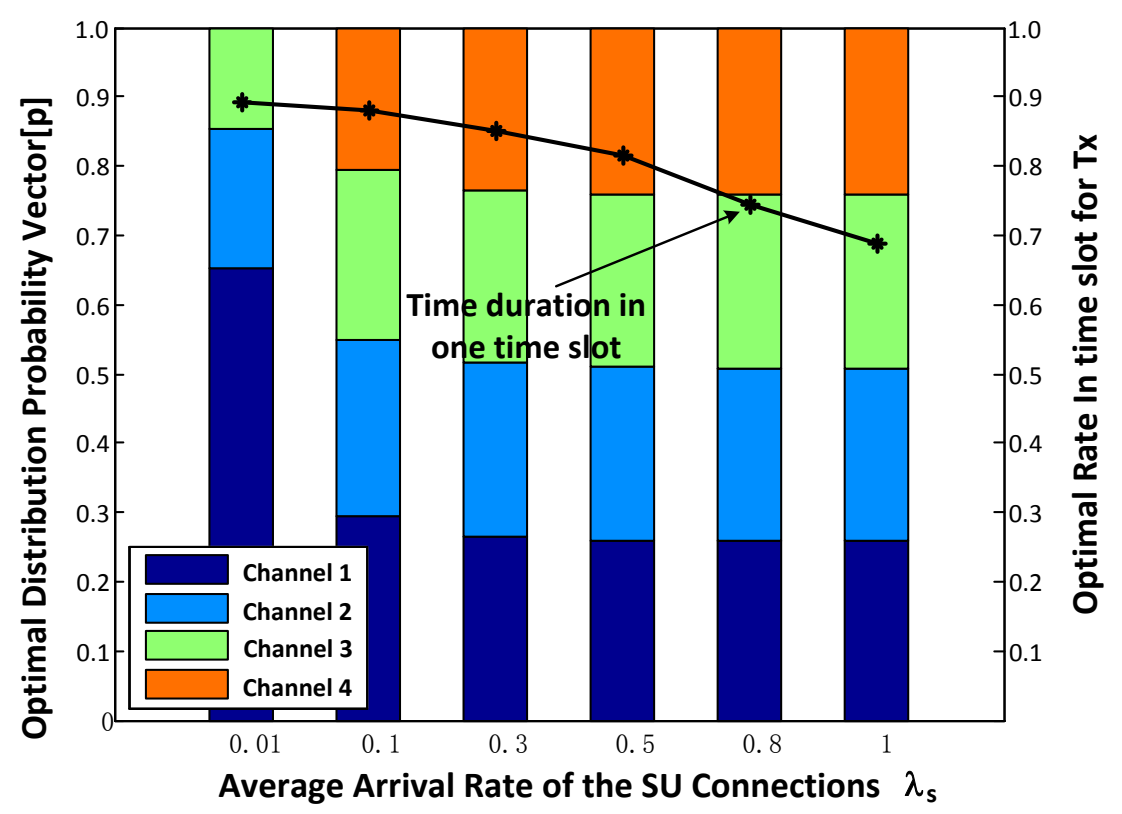

Figure 2. SU traffic pattern with different average arrival rates

Recall that the cell has been separated into $\mathrm{N}$ concentric rings as shown graphically in Fig. 1. When a SU message needs to be transmitted, firstly, it will be placed into a specialized queue for corresponding ring. Secondly, the system of queue is sensing for the available 
vacant frequency channels that which the PU connections do not occupy and tries to distribute the message from SU queue into the candidate channels. We assume without loss of generality that all SU messages have the same data length. As a result, the SU connections in all of the frequency channels are assumed to have the same service time. From the above discussion, it is possible to estimate the optimal time duration for SU traffic in a specific time slot. Then the problem can be simplified into a situation that only SU messages compete for all of the frequency channels, i.e., without competing with the PU connections. Under the above defined framework, the central focus in the sequel is to calculate the number of SU that can be accepted in the different co-eccentric rings in the network under the constraints of service delay elasticity and the blocking probability.

\subsection{Optimal Number of SUs}

In this section, we turn our focus on the SU message competition without competing PU connections. Fig. 3 gives an example of the physical queues for the case of $\mathrm{K}$ frequency channels and $\mathrm{N}$ concentric rings with different modulation and coding schemes. When the traffic of the SUs needs to be transmitted in the system, it can be inputted to the queue for the SU connections. This proposed channel selection model could approximate the virtual SU message queue using an $\mathrm{M} / \mathrm{M} / \mathrm{K} / \mathrm{L}$ queuing system. If the number of SUs is large, the input traffic of the virtual queue can be modeled as a Poisson process, where $\mathrm{K}$ is the number of servers and $\mathrm{L}$ is the finite number of waiting positions for each queue.

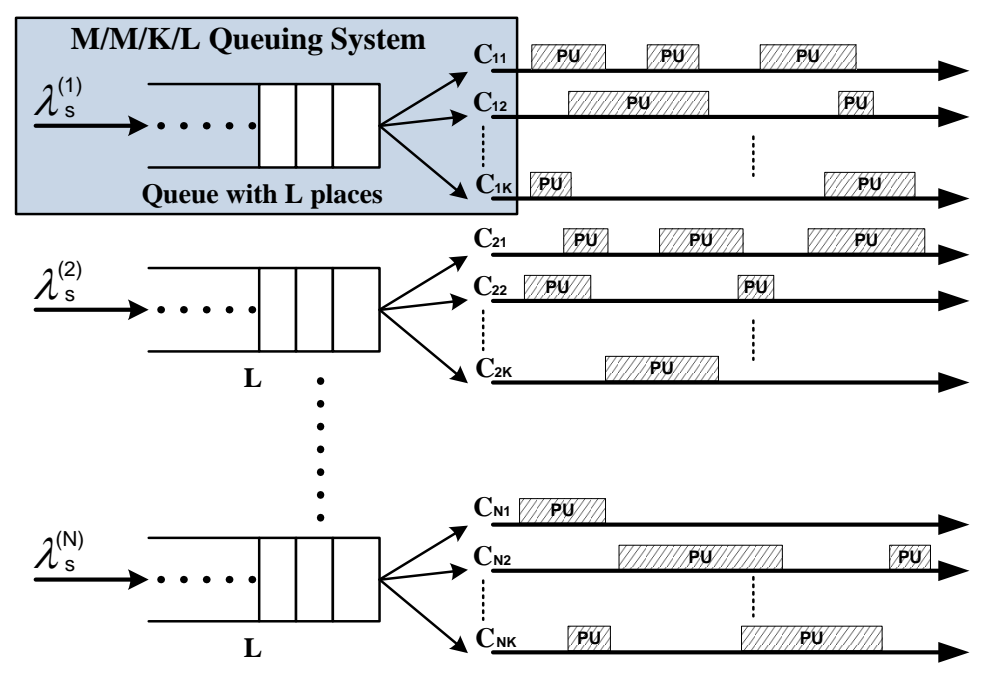

Figure 3. Access for SUs modelled an M/M/K/L queuing system

Let $\lambda_{s}^{(i)}$ denote the average number of the SUs per unit time in ith concentric ring of radii $R_{i}$ and $\mathrm{L}$ denote the number of unit time as a kind of queue length. Therefore, each queue of this system, namely each ring, can accommodate $\lambda_{s}^{(i)} L$ number of the SUs. Given the set of candidate channels $\Omega=\{1,2, \cdots, K\}$ and the set of concentric rings $\Re=\{1,2, \cdots, N\}$, we denote $\mathrm{C}_{\mathrm{ij}}$ to be the capability of the SUs in ring $i \in \mathfrak{R}$ within the channel $j \in \Omega$ and have 


$$
C_{i j}=I E C\left(r_{B S}\right) \frac{B}{K \cdot N}(\mathrm{bit} / \mathrm{s})
$$

where $\mathrm{F}$ is the size of SU message and B represents the bandwidth available at the BS. Let $\mu_{i j}$ represent the service rate of SU connections using the frequency channel $\mathrm{j}$ in ith ring, we have,

$$
\mu_{i j}=\frac{C_{i j}}{F}
$$

Let $\rho_{i}$ denote the occupation rate (offered traffic load), we have

$$
\rho_{i}=\frac{\lambda_{s}^{(i)}}{K \cdot \mu_{i j}}=\frac{F \cdot \lambda_{s}^{(i)}}{K \cdot C_{i j}}
$$

Assume that $p_{m}$ is the probability that there are $\mathrm{m} \mathrm{SU}$ message in the system and $p_{\text {thres }}$ is the blocked traffic rate threshold, therefore we have

$$
p_{m}=\left\{\begin{array}{lr}
\frac{\rho_{i}^{m}}{m !} \cdot p_{0} & m \leq K \\
\frac{\rho_{i}^{m}}{K !}\left(\frac{\rho_{i}}{K}\right)^{m-K} \cdot p_{0} & K<m \leq \lambda_{s}^{(i)} L+K
\end{array}\right.
$$

subject to:

$$
\begin{aligned}
& \sum_{m=0}^{\lambda_{s}^{(i)} L+K} p_{m}=1 \\
& p_{\left(\lambda_{s}^{(i)} L+K\right)} \leq p_{\text {thres }}
\end{aligned}
$$

Note that the constraint of (17) depicts that the blocking rate of SU connections in the virtual queue should be lower than the predetermined threshold $p_{\text {thres }}$.

\section{Numerical Investigation}

Table 1.System Parameters.

\begin{tabular}{|c|c|c|}
\hline Symbol & Definition & Value \\
\hline $\mathbf{P}_{\mathbf{t x}}$ & Received power threshold & $-52 \mathrm{dBm}$ \\
$\mathbf{v a}$ & Vehicle average speed & $8 \mathrm{~m} / \mathrm{s}$ \\
$\mathbf{B W}$ & Bandwidth & $10 \mathrm{MHz}$ \\
$\mathbf{e}_{\mathbf{t x}}$ & Transmitter electronics consumption & $50 \times 10^{-9} \mathrm{~J} / \mathrm{bit}$ \\
$\mathbf{e}_{\mathbf{r x}}$ & Receiver electronics consumption & $50 \times 10^{-9} \mathrm{~J} / \mathrm{bit}$ \\
\hline
\end{tabular}


The related parameters used in general scenarios where a vehicle is moving towards the BS are summarized in Table 1 and we use the model in [32] to calculate the energy consumption of RF module and electronic circuits. The results of energy consumption were obtained via MATLAB based simulation by focusing on the uplink throughput to serving BS and it is assumed that the radius of the cellular BS to be 1000 meters.

We provide an example of optimal allocation of the PU connections and the blocking rate probabilities of SU connections in Table 2. For simplicity, we assume the channel number K $=4$. Note that in Table 2, the arrival rate and service time of the PU connections in all of the $\mathrm{K}$ channels are not identical. In addition, as shown in the results of optimal distribution probability vector for SU transmission, we can see that increased probabilities of SU connections are allocated to the channels whose traffic load is expected to be less. As a result, the wireless node on the vehicle would always try to select the optimal channel for data transmission and avoid the interruption on the PU transmission. Based on the above discussion, the optimal distribution probability vector for transmission time of vehicle $p_{\text {opt }}$ is $(0.2968,0.2518,0.2465,0.2050)$.

Table 2.Parameters of PU Traffic.

\begin{tabular}{|c|c|c|c|}
\hline $\begin{array}{c}\text { Num of } \\
\text { Channel }\end{array}$ & Arrival rate $\left(\lambda_{p}\right)$ & Service time $\left(E\left[X_{p}\right]\right)$ & $\begin{array}{c}\text { Probability of SU traffic } \\
\left(\mathbf{P}_{\mathbf{o p t}}\right)\end{array}$ \\
\hline $\mathbf{1}$ & 0.01 & 0.5 & 0.2968 \\
$\mathbf{2}$ & 0.01 & 0.75 & 0.2518 \\
$\mathbf{3}$ & 0.02 & 0.5 & 0.2465 \\
$\mathbf{4}$ & 0.02 & 0.625 & 0.2050 \\
\hline
\end{tabular}

Recall that the SUs must monitor all frequency channels to sense the arrival of PU connections. In this model, we assume that the PU traffic load is stable within a short time duration. Therefore, from now on, we use queuing system modeling to analyze the traffic of SU connections without consideration of PU connections. The SU virtual queue senses the frequency channel in an increasing order, from 1th to the Mth channel. When initiating a message transmission, the queues first check the availability of frequency channel 1 . If all of the frequency channels are unavailable, the transmission is blocked. Here, for the SU virtual queue, the availability of one frequency channel means that the channel is not occupied by the PU connections.

Table 3 presents the simulation parameters and results with a blocking traffic threshold $p_{\text {thres }}=0.10$ for the SU queue. It can be clearly seen from the results that when the mobile nodes are moving towards the service BS, the length of time units in each ring becomes less owing to the increasing capability of rings in terms of transmission rate. Moreover, with the decreasing distance between wireless nodes and the BS, the ring could accommodate a larger number of SU messages, that is, the SU virtual queue could accommodate a larger number of SUs simultaneously. 
Table 3.Simulation Results ( $\left.\mathrm{p}_{\text {thres }}=0.10\right)$.

\begin{tabular}{|c|c|c|c|c|}
\hline $\begin{array}{c}\text { Num of } \\
\text { Ring }\end{array}$ & $\begin{array}{c}\text { Num of } \\
\text { time unit }\end{array}$ & Max $\lambda_{s}$ & $\begin{array}{c}\text { Packet length } \mathbf{F} \\
\text { (Mbits) }\end{array}$ & $\begin{array}{c}\text { Blocking } \\
\text { Probability }\end{array}$ \\
\hline $\mathbf{1}$ & 42 & 7 & 1 & 0.0490 \\
$\mathbf{2}$ & 23 & 11 & 1 & 0.0909 \\
$\mathbf{3}$ & 84 & 14 & 1 & 0.0477 \\
$\mathbf{4}$ & 77 & 22 & 1 & 0.0909 \\
$\mathbf{5}$ & 167 & 29 & 1 & 0.0805 \\
$\mathbf{6}$ & 188 & 33 & 1 & 0.0909 \\
total & 581 & 116 & 1 & N/A \\
optimal & 188 & 199 & 1 & 0.0955 \\
\hline
\end{tabular}

In this model, we assume that there are two situation for a comparison in order to show the benefit of energy consumption: 1) the bandwidth of the service BS is divided into six equal parts, and each ring could only use one part for transmission; 2) the bandwidth is occupied exclusively by the last ring which is closest to the service BS. For instance, in the 7th line of table 3, the SUs buffer all the message and move into the area of the ring which is closest to the service BS. In this case, the SUs in the last ring occupy all of the bandwidth for message transmission, that the capacity and throughput will be the maximum possible since this ring can support the higher constellation. From Fig. 4 we can observe that, if the message carried by the SUs is highly delay-tolerant, when the SUs transmit message in the closest ring of the BS, the throughput could be significantly improved.

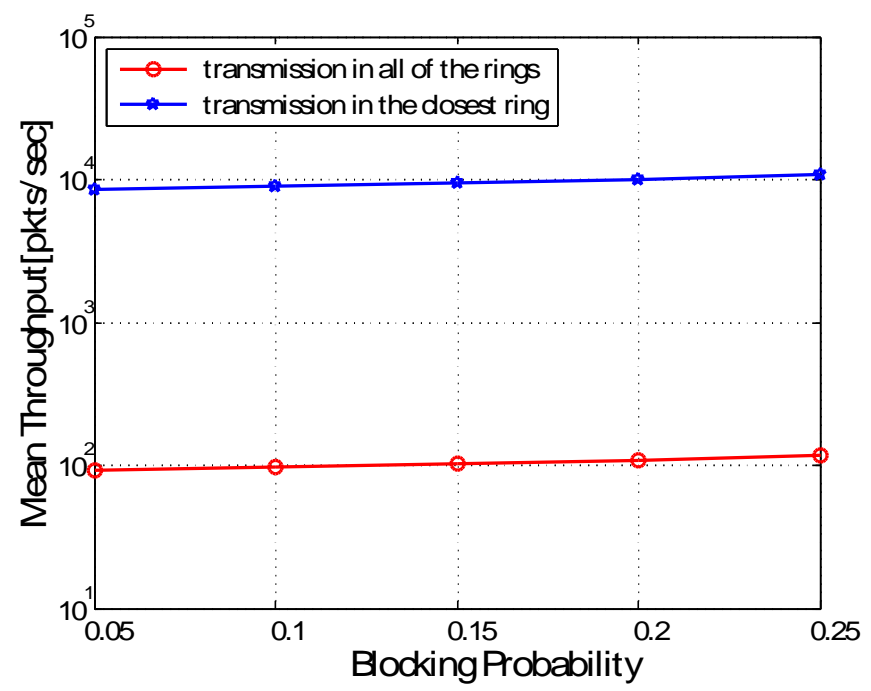

Figure 4. Mean throughput of the SUs vs. different blocking probability threshold

Concerning the energy consumption, Fig. 5 presents three different cases of energy cost focusing on one SU to be served. Firstly, when the wireless node is moving towards the BS, we compute the energy consumption in all the six rings of the BS. A second case shown is the 
situation when the SU buffers the message to be transmitted to the BS and moves into the last ring for data transmission, which the SU occupies all of the bandwidth of the BS. Lastly, and based on the second case, the proposed scheme is depicted where the SU has moved into the last ring of the BS, as the bandwidth increasing, the SU queuing system can accommodate an increased number of SU messages under a threshold of blocking traffic rate, which brings all the capabilities into full play. Here, we must emphasize that in the situation of the first and third case, the queuing system works under an identical blocking probability threshold to restrict the message number in the queue. As a result, intuitively speaking, the optimal one (the third case) has the potential of increasing the energy efficiency due to the fact that it can process a higher number of SU message simultaneously; thus spending less time to transmit the message comparing to the sub-optimal one (considering messages of the same size). From Fig. 5, it is shown that, as the threshold of blocking traffic probability is set to be $10 \%$, the optimal schemes only dissipate approximately $2 / 5$ energy (Joule) with equal amounts of message transmission. It is noteworthy that, the energy cost of optimal schemes fluctuates narrowly among these three schemes under different blocking probability thresholds. The reason is that the optimal schemes provide the highest data throughput and accommodate largest number of SU messages, which leads to the shortest time duration for wireless transmission and circuit cost.

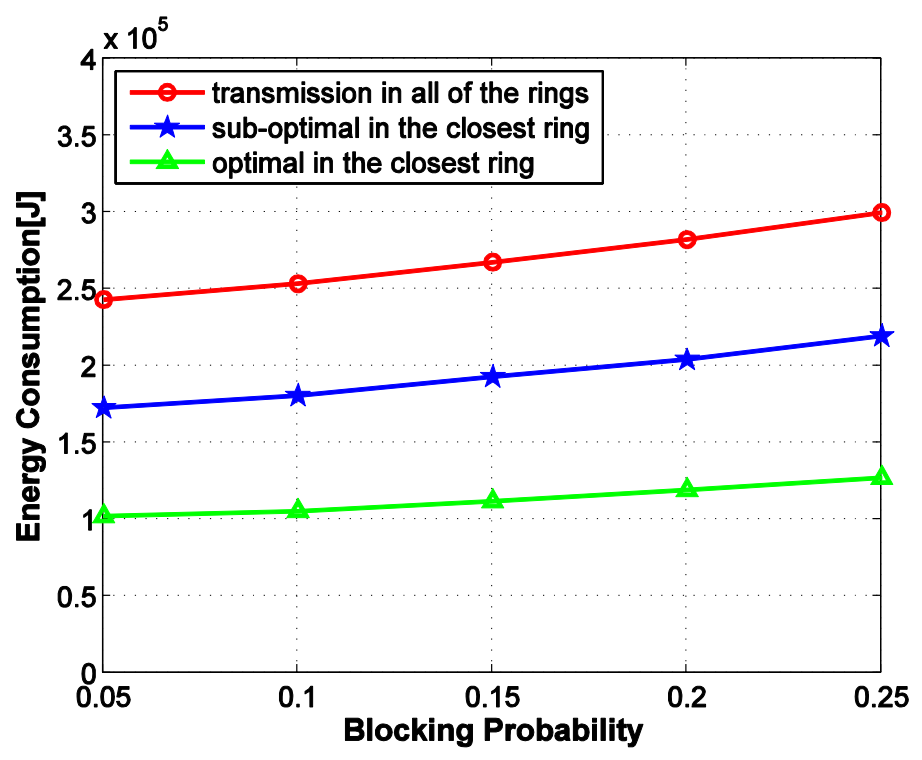

Figure 5. Energy consumption of transmission in different blocking probability thresholds for one SU

It is assumed that the length of each message is identical. Due to the different throughput of wireless transmission in different rings of area, the length of time units that the wireless nodes could send one message to BS should be different as shown in Fig. 6. Meanwhile, with the monotonically increasing values of blocking probability threshold, the number of SU message that can be accommodated in the queuing systems will rise in varying degrees. Compared to the first two cases, the optimal schemes can accommodate significantly more messages in the queuing systems simultaneously as shown in Fig. 7. 


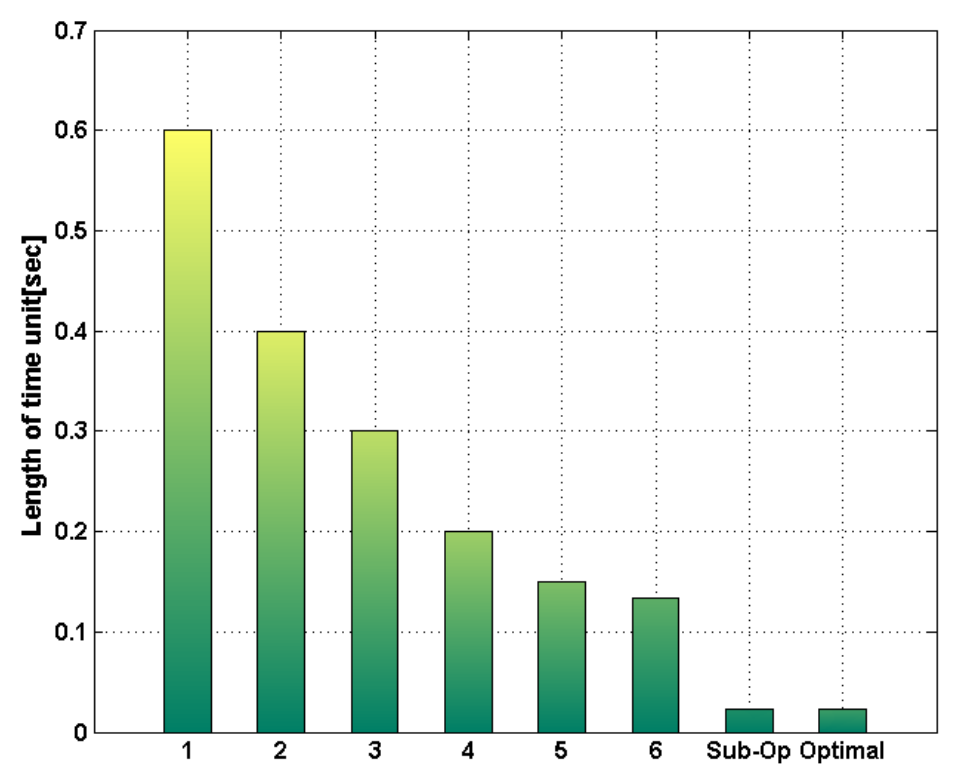

Figure 6. The length of time unit in different rings

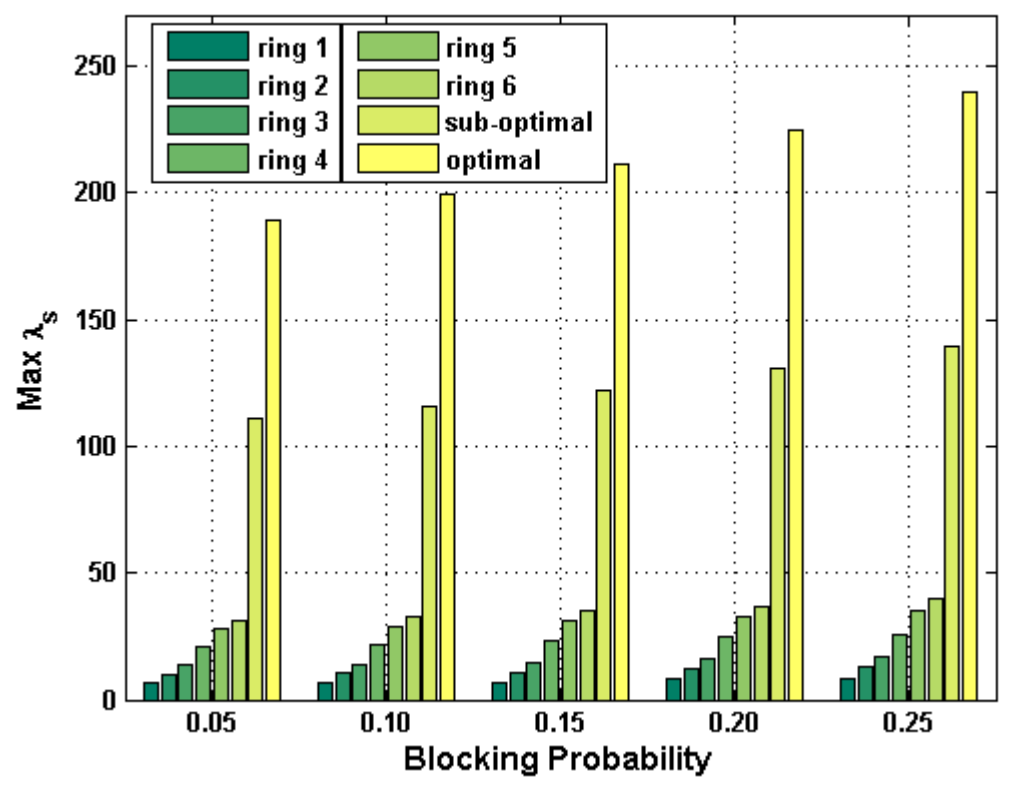

Figure 7. The max value of_s in different rings under different blocking probability thresholds

Additionally, the scenario investigated above can be extended for the case where there are multiple SUs transmitting messages simultaneously. For illustration purposes, we set a blocking traffic threshold $p_{\text {thres }}=0.10$ for the SU queues in this simulation as well. As presented in Fig. 8, the trends of three curves are almost the same as before and the proposed scheme outperforms the other schemes. 


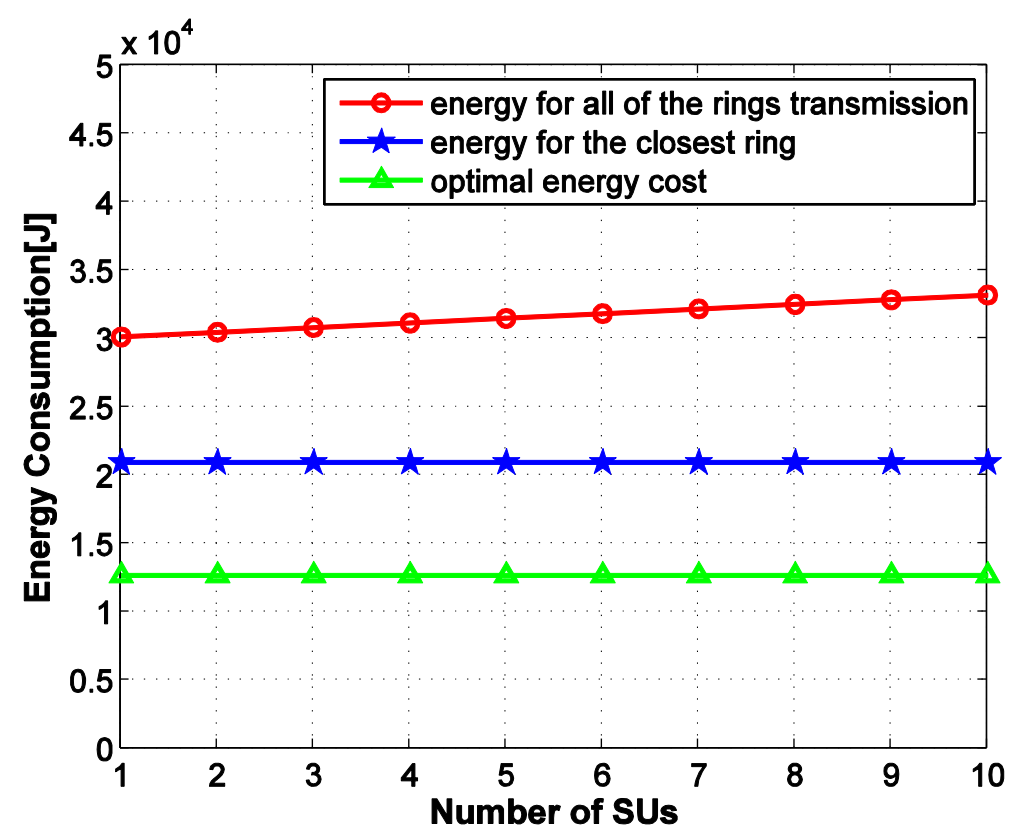

Figure 8. Energy consumption of transmission with increasing SU number

\section{Final Remarks and Conclusions}

In this paper, a scheme is proposed for delay-tolerant Internet applications in CR networks with the central aim of reducing the energy consumption; and as a result to prolong the recharging periods of terminals. Considering the stochastic distribution of the PU arrival rate and time duration in primary channels, the SUs has to query a trusted database for historical information in order to select candidate channel for message transmission. With the aid of the estimated available probability concerning location and time duration, the SU traffic can access the primary channels more efficiently and increase the channel utilization. Furthermore, we took the $\mathrm{M} / \mathrm{M} / \mathrm{K} / \mathrm{L}$ queue to derive the average number of simultaneous $\mathrm{SU}$ message and corresponding blocking probabilities for different situations. When non-real-time applications are buffered in the SU systems, our optimal schemes have an inclination to delay the message transmission to the area with higher throughput, i.e., closer to the BS. Our analysis and numerical results reveal that the proposed optimal message transmission schemes can accommodate significantly more messages in the queue simultaneously under the same blocking threshold. Also, due to the highest data throughput of the proposed schemes, the overall energy cost of message transmission can also be significantly decreased. Finally, when the scenario is extended to multiple SUs cases, the proposed scheme still outperforms the other schemes. In the future, once the details of database regarding PU traffic is available for practical use, we plan to study in a more detailed manner the technique that uses the available gaps in radio spectrum, (the so-called 'white spaces'), which exist in the bands that have been reserved for analog TV broadcasting. 


\section{References}

[1] Simon Haykin, "Cognitive Radio: Brain-Empowered Wireless Communications", IEEE Journal on Selected Areas in Communications, vol.23, no. 2, pp. 201-220, February 2005. http://dx.doi.org/10.1109/JSAC.2004.839380

[2] Ian F. Akyildiz, Won-Yeol Lee, Mehmet C. Vuran, and Shantidev Mohanty, "NeXt generation/dynamic spectrum access/cognitive radio wireless networks: a survey", Computer Networks: The International Journal of Computer and Telecommunications Networking, vol. 50, no. 13, pp. 2127-2159, Sept. 2006. http://dx.doi.org/10.1016/j.comnet.2006.05.001

[3] Xiukui Li and Seyed A. Zekavat, "Traffic Pattern Prediction and Performance Investigation for Cognitive Radio Systems", Wireless Communications and Networking Conference (WCNC), pp. 894-899, Mar. 2008. http://dx.doi.org/10.1109/WCNC.2008.163

[4] Qianchuan Zhao, Stefan Geirhofer, Lang Tong and Brian M. Sadler, "Opportunistic Spectrum Access via Periodic Channel Sensing”, IEEE Transactions on Signal Processing, vol. 56, no. 2 pp. 785-796, Feb. 2008. http://dx.doi.org/10.1109/TSP.2007.907867

[5] Gosan Noh, Jemin Lee and Daesik Hong, "Stochastic Multichannel Sensing for Cognitive Radio Systems: Optimal Channel Selection for Sensing with Interference Constraints", Vehicular Technology Conference Fall (VTC 2009-Fall), pp. 1-5, Sept. 2009. http://dx.doi.org/10.1109/VETECF.2009.5379040

[6] Peter J. Smith, Abdulla Firag, Pawel A. Dmochowski and Mansoor Shafi, "Analysis of the M/M/N/N Queue with Two Types of Arrival Process: Applications to Future Mobile Radio Systems", Journal of Applied Mathematics, 2012. http://dx.doi.org/10.1155/2012/123808

[7] Junghyun Heo, and Jungchae Shin et al., "Mathematical Analysis of Secondary User Traffic in Cognitive Radio System", Vehicular Technology Conference 2008 (VTC 2008-Fall), pp. 1-5, Sept. 2008. http://dx.doi.org/10.1109/VETECF.2008.266

[8] Shanshan Wang, Junshan Zhang and Lang Tong, "A Characterization of Delay Performance of Cognitive Medium Access", IEEE Transactions on Wireless Communications, vol. 11, no. 2, pp. 800-809, Feb. 2012. http://dx.doi.org/10.1109/TWC.2012.010312.110765

[9] Hsien-Po Shiang and Mihaela van der Schaar, "Queuing-Based Dynamic Channel Selection for Heterogeneous Multimedia Applications Over Cognitive Radio Networks", IEEE Transactions on Multimedia, vol. 10, no. 5, pp. 896-909, Aug. 2008. http://dx.doi.org/10.1109/TMM.2008.922851

[10] Stephen J. Shellhammer, Ahmed K. Sadek and Wenyi Zhang, "Technical challenges for cognitive radio in the TV white space spectrum", Information Theory and Applications Workshop, pp.323-333, Feb. 2009. http://dx.doi.org/10.1109/ITA.2009.5044964

[11]S. Probasco, Ed. B. Patil, "Protocol to Access White Space database: PS, use cases and rqmts", online at: http://tools.ietf.org/html/draftietf-paws-problem-stmt-usecases-rqmts-03.

[12]E. Uysal-Biyikoglu, B. Prabhakar, and A. El Gamal, "Energy-efficient packet transmission over a wireless link", IEEE/ACM Transactions on Networking, vol. 10, no. 4, 
pp. 487-499, 2002. http://dx.doi.org/10.1109/TNET.2002.801419

[13]Xiliang Zhong, and Cheng-Zhong Xu, "Delay-Constrained Energy-Efficient Wireless Packet Scheduling with QoS Guarantees”, GLOBECOM 2005, vol. 6, pp. 3336-3340, 2005. http://dx.doi.org/10.1109/GLOCOM.2005.1578392

[14] Alvin Fu, Eytan Modiano, and John Tsitsiklis, "Optimal Energy Allocation for Delay-Constrained Data Transmission over a Time-Varying Channel”, INFOCOM 2003, vol. 2, pp. 1095-1105, July 2003. http://dx.doi.org/10.1109/INFCOM.2003.1208946

[15]Hongseok Kim, and Gustavo de Veciana, "Leveraging dynamic spare capacity in wireless systems to conserve mobile terminals' energy", IEEE/ACM Transactions on Networking (TON), vol. 18, no. 3, pp. 802-815, June 2010. http://dx.doi.org/10.1109/TNET.2009.2032238

[16] Wanshi Chen, Michael J. Neely, and Urbashi Mitra, "Energy-Efficient Transmissions With Individual Packet Delay Constraints", IEEE Transactions on Information Theory, vol. 54, no. 5, 2090-2109, May 2008. http://dx.doi.org/10.1109/TIT.2008.920344

[17] Bo Han, et al., "Cellular traffic offloading through opportunistic communications: a case study", Proceedings of the 5th ACM workshop on Challenged networks (CHANTS '10), New York, NY, USA, 2010. http://dx.doi.org/10.1145/1859934.1859943

[18]Xuejun Zhuo, Wei Gao, Guohong Cao, and Yiqi Dai, "Win-Coupon: An incentive framework for $3 \mathrm{G}$ traffic offloading”, Network Protocols (ICNP), pp. 206-215, Vancouver, BC, Oct. 2011. http://dx.doi.org/10.1109/ICNP.2011.6089054

[19] Simon Fischer, Petri Mahonen, Marcel Schongens, and Berthold Vocking, "Load Balancing for Dynamic Spectrum Assignment with Local Information for Secondary Users", New Frontiers in Dynamic Spectrum Access Networks (DySPAN 2008), Chicago, USA, pp. 1-9, Oct. 2008. http://dx.doi.org/10.1109/DYSPAN.2008.38

[20]Li-Chun Wang, et al., "Load-Balancing Spectrum Decision for Cognitive Radio Networks", IEEE Journal on Selected Areas in Communications, vol. 29, no. 4, pp. 757-769, April 2011. http://dx.doi.org/10.1109/JSAC.2011.110408

[21] Qinghai Xiao, Yunzhou Li, Ming Zhao, Shidong Zhou, and Jing Wang, "Opportunistic channel selection approach under collision probability constraint in cognitive radio systems", Computer Communications, vol. 32, no. 18, pp. 1914-1922, Dec. 2009. http://dx.doi.org/10.1016/j.comcom.2009.06.015

[22] Guangxiang Yuan, Ryan C. Grammenos, Yang Yang, and Wenbo Wang, "Performance Analysis of Selective Opportunistic Spectrum Access With Traffic Prediction", IEEE Transactions on Vehicular Technology, vol. 59, no. 4, pp. 1949-1959, MAY 2010. http://dx.doi.org/10.1109/TVT.2009.2039155

[23] Johanna Vartiainen, Marko Hoyhtya, Janne Lehtomaki and Timo Braysy, "Priority Channel Selection Based on Detection History Database", 2010 Proceedings of the Fifth International Conference on Cognitive Radio Oriented Wireless Networks \& Communications (CROWNCOM), Cannes, pp. 1-5, June 2010. 
[24]Ho Ting Cheng, and Weihua Zhuang, "Simple Channel Sensing Order in Cognitive Radio Networks", IEEE Journal on Selected Areas in Communications, vol. 29, no. 4, pp. 676-688, APRIL 2011. http://dx.doi.org/10.1109/JSAC.2011.110402

[25] Osvaldo Simeone, Yeheskel Bar-Ness, and Umberto Spagnolini, "Stable Throughput of Cognitive Radios With and Without Relaying Capability", IEEE Transactions on Communications, vol. 55, no. 12, pp. 2351-2360, Dec. 2007. http://dx.doi.org/10.1109/TCOMM.2007.910699

[26]Chung-Wei Wang, and Li-Chun Wang, "Analysis of Reactive Spectrum Handoff in Cognitive Radio Networks", IEEE Journal on Selected Areas in Communications, vol. 30, no. 10, pp. 2016-2028, NOVEMBER 2012. http://dx.doi.org/10.1109/JSAC.2012.121116

[27] Caoxie Zhang, Xinbing Wang, and Jun Li, "Cooperative Cognitive Radio with Priority Queueing Analysis”, IEEE International Conference on Communications (ICC'09), Dresden, pp. 1-5, June 2009. http://dx.doi.org/10.1109/ICC.2009.5198858

[28] Chittabrata Ghosh, Srikanth Pagadarai, Dharma P. Agrawal, and Alexander M. Wyglinski, "Queueing Theory Representation and Modeling of Spectrum Occupancy Employing Radio Frequency Measurements", 2009 IEEE 70th Vehicular Technology Conference Fall (VTC 2009 Fall), Anchorage, AK, pp. $\quad 1-5, \quad$ Sept. 2009. http://dx.doi.org/10.1109/VETECF.2009.5379051

[29] Oussama Habachi, and Yezekael Hayel, “Optimal Sensing Strategy for Opportunistic Secondary Users In a Cognitive Radio Network", Proceedings of the 13th ACM international conference on Modeling, analysis, and simulation of wireless and mobile systems (MSWIM '10), Bodrum, Turkey, pp. 343-350, 010. http://dx.doi.org/10.1145/1868521.1868577

[30] Rainer Schoenen and Bernhard H. Walke, "On PHY and MAC performance of 3G-LTE in a multi-hop cellular environment", Wireless Communications, Networking and Mobile Computing (WiCom) 2007, Shanghai, China, pp. 926-929, Sept. 2007. http://dx.doi.org/ 10.1109/WICOM.2007.238

[31]Bi Zhao, and Vasilis Friderikos, "Optimal Stopping for Energy Efficiency with Delay Constraints in Cognitive Radio Networks", IEEE 23rd International Symposium on Personal, Indoor and Mobile Radio Communications (PIMRC 2012), pp. 820-825, Sydney, Australia, Sept. 2012. http://dx.doi.org/10.1109/PIMRC.2012.6362897

[32]P. Kolios, V. Friderikos and K. Papadaki, "Ultra low energy store-carry and forward relaying within the cell", IEEE Vehicular Technology Conference, pp. 1-5, Sept. 2009. http://dx.doi.org/10.1109/VETECF.2009.5379030

\section{Copyright Disclaimer}

Copyright reserved by the author(s).

This article is an open-access article distributed under the terms and conditions of the Creative Commons Attribution license (http://creativecommons.org/licenses/by/3.0/). 